\title{
ON THE DISTRIBUTION OF RAMIFICATION POINTS IN TRIGONAL CURVES
}

\author{
CÍCERO F. CARVALHO
}

(Received 5 June 1998)

\begin{abstract}
We study the distribution of the total and ordinary ramification points of a trigonal curve over the intersection of this curve with rational curves on a rational normal scroll. We show, among other results, that these intersections may contain all the ramification points of the trigonal curve.
\end{abstract}

Keywords and phrases. Trigonal curves, ramification points, curves on surfaces.

1991 Mathematics Subject Classification. 14H30, 14H45.

1. Introduction. Trigonal curves are canonically immersed in rational normal scrolls, which may be viewed as the join of two rational normal curves lying in hyperplanes of complementary dimension. The purpose of this note is to find relations and bounds for the number of total and ordinary ramification points of a trigonal curve that lie on the intersection with a rational curve on the scroll. The motivation comes from results obtained by Coppens [2] relating the number of total ramification points having one or other Weierstrass sequence in a trigonal curve. In a more geometrical approach to that question, Stöhr and Viana [4] showed that a ramification point have one or other Weierstrass sequence depending on whether or not it lies on a certain rational curve (the directrix of the scroll). We use this same approach in what follows. This simplifies the treatment of the question and adds a geometrical meaning to the results (cf. [3]).

2. Preliminaries. Let $C$ be a nonsingular trigonal curve of genus $g \geq 5$ defined over an algebraically closed field $k$ of a characteristic zero and canonically embedded in $\mathbb{P}^{g-1}(k)$. We know from [1] that such a curve lies in a rational normal scroll $S_{m n}$ that may be described as the join of the rational normal curves

$$
D:=\left\{\left(a^{n}: a^{n-1} b: \cdots: b^{n}: 0: \cdots: 0\right) \in \mathbb{P}^{m+n+1}(k) \mid(a: b) \in \mathbb{P}^{1}(k)\right\}
$$

and

$$
E:=\left\{\left(0: \cdots: 0: a^{m}: a^{m-1} b: \cdots: b^{m}\right) \in \mathbb{P}^{m+n+1}(k) \mid(a: b) \in \mathbb{P}^{1}(k)\right\},
$$

where $m$ and $n$ are positive integers satisfying $m \leq n,(n-2) / 2 \leq m \leq 2 n+2$ and $m+n+2=g$.

We denote the lines of the ruling by

$$
L_{b / a}:=\overline{\left(a^{n}: a^{n-1} b: \cdots: b^{n}: 0: \cdots: 0\right),\left(0: \cdots: 0: a^{m}: a^{m-1} b: \cdots: b^{m}\right)}
$$

with $b / a \in \mathbb{P}^{1}(k)=k \cup\{\infty\}$ and we observe that the following sets form an open 
covering for $S_{m n}$.

$$
\begin{aligned}
U_{0} & :=S_{m n} \backslash\left(L_{\infty} \cup E\right)=\left\{\left(a^{0}: \cdots: a^{n}: a^{0} b: \cdots: a^{m} b\right) \mid(a, b) \in k^{2}\right\}, \\
U_{n} & :=S_{m n} \backslash\left(L_{0} \cup E\right)=\left\{\left(a^{n}: \cdots: a^{0}: a^{m} b: \cdots: a^{0} b\right) \mid(a, b) \in k^{2}\right\}, \\
U_{n+1} & :=S_{m n} \backslash\left(L_{\infty} \cup D\right)=\left\{\left(a^{0} b: \cdots: a^{n} b: a^{0}: \cdots: a^{m}\right) \mid(a, b) \in k^{2}\right\}, \\
U_{g-1} & :=S_{m n} \backslash\left(L_{0} \cup D\right)=\left\{\left(a^{n} b: \cdots: a^{0} b: \cdots: a^{m}: \cdots: a^{0}\right) \mid(a, b) \in k^{2}\right\} .
\end{aligned}
$$

Note that each of these sets is isomorphic to the affine plane $k^{2}$ and, in [4], the following theorem was proved.

THEOREM [1, Thm. 1.1]. Associating to each plane curve the Zariski closure of its image under the local parametrization $k^{2} \hookrightarrow S_{m n}$, defined by

$$
(a, b) \longmapsto\left(a^{0}: \cdots: a^{n}: a^{0} b: \cdots: a^{m} b\right),
$$

one obtains a bijective correspondence between the canonical curves on the scroll $S_{m n} \subset$ $\mathbb{P}^{m+n+1}(k)$ and the affine plane curves given by the irreducible equation

$$
c_{3}(X) Y^{3}+c_{2}(X) Y^{2}+c_{1}(X) Y+c_{0}(X)=0
$$

satisfying $c_{3}(X) \neq 0, \operatorname{deg} c_{i}(X) \leq d_{i}$ for each $i=0,1,2,3$ and $\operatorname{deg} c_{i}(X)=d_{i}$ for at least one $i$, where $d_{0}=2 n-m+2, d_{1}=n+2, d_{2}=m+2$, and $d_{3}=2 m-n+2$.

In what follows, we always denote an equation as the above by $f_{0}(X, Y)=0$ and we call $C_{0}$ the plane curve defined by it. Observe that if $C$ is the correspondent trigonal curve in $S_{m n}$, then $C_{0}$ is isomorphic to the affine curve $C \cap U_{0}$. Furthermore, there is a plane curve which we call $C_{n}$ that is isomorphic to $C \cap U_{n}$ under the local parametrization of $S_{m n}$ defined by $(a: b) \mapsto\left(a^{n}: \cdots: a^{0}: a^{m} b: \cdots: a^{0} b\right)$. Denoting by $f_{n}(X, Y)=0$ the equation of $C_{n}$, we must have $f_{n}(X, Y)=\tilde{c}_{3}(X) Y^{3}+\tilde{c}_{2}(X) Y^{2}+$ $\tilde{c}_{1}(X) Y+\tilde{c}_{0}(X)$, where $\tilde{c}_{i}(X):=c_{i}(1 / X) X^{(2 n-m+2-i(n-m))}, i=0,1,2,3$ (to see that, observe that if $\left(a^{0}: \cdots: a^{n}: a^{0} b: \cdots: a^{m} b\right)=\left(\tilde{a}^{n}: \cdots: \tilde{a}^{0}: \tilde{a}^{m} \tilde{b}: \cdots: \tilde{a}^{0} \tilde{b}\right)$, then $\tilde{a}=1 / a$ and $\left.\tilde{b}=b / a^{n-m}\right)$. Similarly, we denote by $f_{n+1}(X, Y)=0$ the equation of the plane curve $C_{n+1}$ which is isomorphic to the affine curve $C \cap U_{n+1}$ through the local parametrization of $S_{m n}$, defined by $(a, b) \mapsto\left(a^{0} b: \cdots: a^{n} b: a^{0}: \cdots: a^{m}\right)$, and as above we may conclude that $f_{n+1}(X, Y)=c_{0}(X) Y^{3}+c_{1}(X) Y^{2}+c_{2}(X) Y+c_{3}(X)$. Finally, we denote by $f_{g-1}(X, Y)=0$ the equation of the plane curve $C_{g-1}$ which is isomorphic to the affine curve $C \cap U_{g-1}$ through the local parametrization of $S_{m n}$, defined by $(a, b) \mapsto\left(a^{n} b: \cdots: a^{0} b: a^{0}: \cdots: a^{m}\right)$, and one may easily verify that $f_{g-1}(X, Y)=\tilde{c}_{0}(X) Y^{3}+\tilde{c}_{1}(X) Y^{2}+\tilde{c}_{2}(X) Y+\tilde{c}_{3}(X)$.

A point $P \in C$ is a ramification point with respect to the trigonal morphism of $C$ if the line $L$ of the scroll passing through $P$ is the tangent line at $P$, and the ramification index $e_{P}$ is the intersection multiplicity of $C$ and $L$ at $P$. We say that $P$ is a total ramification point (respectively, an ordinary ramification point) when $e_{P}=3$ (respectively, when $e_{P}=2$ ). Observe that maps $k^{2} \hookrightarrow U_{0}$ and $k^{2} \hookrightarrow U_{n+1}$ (respectively, $k^{2} \hookrightarrow U_{n}$ and $k^{2} \hookrightarrow U_{g-1}$ ) take the vertical line $X=a$ of $k^{2}$ into $L_{a}$ (respectively, into $L_{1 / a}$ ), $a \in k$. Thus, we call a point of $C_{i}(i=0, n, n+1, g-1)$ a ramification point if it has a vertical tangent line and we classify it as a total ramification point or an ordinary ramification 
point according to its intersection multiplicity with the tangent line as above. Equivalently, a point $P=(a, b) \in C_{i}(i=0, n, n+1, g-1)$ is a total (respectively, an ordinary) ramification point if and only if $\operatorname{ord}_{P}(x-a)=3$ (respectively, $\operatorname{ord}_{P}(x-a)=2$ ), where $x$ is the rational function of $k\left(C_{i}\right)=k(C)$, defined by $(a, b) \rightarrow a$, and $\operatorname{ord}_{P}(h)$ is the order at $P$ of the rational function $h \in k\left(C_{i}\right)$.

We end this section with the following observations.

REMARK 2.1. It is easy to check the following facts:

(i) If $(a, b)$ is a total ramification point of $C_{0}$, then $c_{3}(a) \neq 0$.

(ii) A point $(a, 0) \in k^{2}$ is a total (respectively, an ordinary) ramification point of $C_{0}$ if and only if $c_{0}(a)=0, c_{1}(a)=0$ and $c_{2}(a)=0$ (respectively, $c_{2}(a) \neq 0$ ).

(iii) A point $(a, 0) \in k^{2}$ is a total (respectively, an ordinary) ramification point of $C_{n}$ if and only if $\tilde{c}_{0}(a)=0, \tilde{c}_{1}(a)=0$ and $\tilde{c}_{2}(a)=0$ (respectively, $\tilde{c}_{2}(a) \neq 0$ ).

(iv) A point $(a, 0) \in k^{2}$ is a total (respectively, an ordinary) ramification point of $C_{n+1}$ if and only if $c_{3}(a)=0, c_{2}(a)=0$ and $c_{1}(a)=0$ (respectively, $c_{1}(a) \neq 0$ ).

(v) The point $(a, 0) \in k^{2}$ is a total (respectively, an ordinary) ramification point of $C_{g-1}$ if and only if $\tilde{c}_{3}(a)=0, \tilde{c}_{2}(a)=0$ and $\tilde{c}_{1}(a)=0$ (respectively, $\tilde{c}_{1}(a) \neq 0$ ).

REMARK 2.2. If $\operatorname{deg} c_{3}(X) \leq 2 m-n$, then $X^{2} \mid \tilde{c}_{3}(X)$ and $(0,0) \in C_{g-1}$. Since this curve is nonsingular, we must have $X \nmid \tilde{c}_{2}(X)$, i.e., $\operatorname{deg} c_{2}(X)=m+2$. Similarly, if $\operatorname{deg} c_{0}(X) \leq 2 n-m$, then we must have $\operatorname{deg} c_{1}(X)=n+2$.

REMARK 2.3. If $(a, 0) \in k^{2}$ is a ramification point of $C_{n+1}$, then $a$ is a simple root of $c_{3}(X)$. In fact, if $a$ is a multiple root of $c_{3}(X)$ and also a root of $c_{2}(X)$, then $(a, 0)$ is a singular point of $C_{n+1}$, which is absurd. Similarly, if $(a, 0) \in k^{2}$ is a ramification point of $C_{0}$, then $a$ is a simple root of $c_{0}(X)$.

3. The main results. The only rational curves on $S_{m n}$ are the lines of the ruling and the intersection of the scroll with the hyperplanes of $\mathbb{P}^{m+n-1}(k)$. We want to find bounds for the number of ramified points that lie on the intersection of a trigonal curve on the scroll with rational curves of the latter type. When $m=n$, all such rational curves are linearly equivalent as divisors on $S_{m n}$ and after a change of variables, it is enough to consider the points on $C \cap D$. When $m<n$ though, $E$ is a distinguished curve on the scroll (e.g., is the only curve with negative self-intersection) and then we must also consider the points on $C \cap E$ (in this case, $E$ is called the directrix of the scroll).

To study the occurrence of ramification points on the intersections $C \cap D$ and $C \cap E$, we use the local parametrizations and the curves $C_{0}, C_{n}, C_{n+1}$, and $C_{g-1}$ introduced above. We see that in order to count such points, it suffices to count the number of ramification points of zero ordinate in $C_{0}$ and in $C_{n+1}$, then the number of ramification points with zero abscissa on $C_{n}$, and finally to check if the origin of the plane containing $C_{g-1}$ is a ramification point of that curve.

Proposition 3.1. Let $\sigma$ (respectively, $\rho$ ) denote the number of total (respectively, ordinary) ramification points of $C$ lying over $E$ and let $\eta$ (respectively, $\xi$ ) denote the number of total (respectively, ordinary) ramification points of C lying over D. Then one of the following possibilities occurs: 
(i) $0 \leq \sigma+\rho \leq 2 m-n+2,0 \leq \sigma+\rho+\eta \leq m+2,0 \leq \eta+\xi+\sigma \leq n+2$.

(ii) $\sigma+\rho=2 m-n+2, \xi=0$ and $n-m+1 \leq \eta \leq n+2-\sigma$.

(iii) $\rho=0, \xi+\eta=2 n-m+2,0 \leq \sigma \leq 2 m-n+2$ and $0 \leq \sigma+\eta \leq m+2$.

(iv) $\sigma=2 m-n+2, \eta=2 n-m+2$ and $\rho=\xi=0$.

In the case $m<n$, the above possibilities are mutually exclusive.

Proof. Suppose that $c_{2} \neq 0$ and $c_{1} \neq 0$. If there is a total ramification point in $C \cap\left(D \backslash L_{\infty}\right)$, then Remark 2.1(ii) implies that, for some $b \in k$, we have $(X-b) \mid c_{0}(X)$, $(X-b) \mid c_{1}(X)$ and $(X-b) \mid c_{2}(X)$. From Remarks 2.2 and 2.1(iii), we have that $D \cap L_{\infty}$ is a total (respectively, an ordinary) ramification point of $C$ if and only if $\operatorname{deg} c_{0}(X)=$ $2 n-m+1, \operatorname{deg} c_{1}(X) \leq n+1$, and $\operatorname{deg} c_{2}(X) \leq m+1$ (respectively, $\operatorname{deg} c_{2}(X)=m+2$ ). From all this, we may conclude that $0 \leq \sigma+\rho \leq 2 m-n+2$. The other inequalities in (i) follow similarly.

To establish the inequalities in (ii), we suppose that $c_{2}(X)=0$ and $c_{1}(X) \neq 0$. Then by Remark 2.1(iv), the roots of $c_{3}(X)$ are in a one-to-one correspondence with the ramification points of $C_{n+1}$ of the form $(a, 0)$, with $a \in k$ (i.e., the roots of $c_{3}(X)$ are in a one-to-one correspondence with the ramification points of $\left.C \cap\left(E \backslash L_{\infty}\right)\right)$. From Remark 2.3, it follows that $c_{3}(X)$ does not have a multiple root. By Remark 2.2, since $c_{2}(X)=0$, we must have $\operatorname{deg} c_{3}(X)=2 m-n+2$ or $\operatorname{deg} c_{3}(X)=2 m-n+1$, but either way we have $\sigma+\rho=2 m-n+2$. Obviously, we have $\xi=0$ and $0 \leq \eta+\sigma \leq n+2$. Now, we do not have the restriction $0 \leq \sigma+\rho+\eta \leq m+2$ and to avoid an overlapping with the conditions in (i), we take $\eta>m+2-(\sigma+\rho)=n-m$ and, thus, $n-m+1 \leq \eta \leq$ $n+2-\sigma$.

Using Remarks 2.1, 2.2, and 2.3 in the same way as above, one may prove (iii) and (iv), the former by assuming $c_{1}(X)=0$, and $c_{2} \neq 0$ and the latter by assuming $c_{1}(X)=$ $c_{2}(X)=0$.

We will show constructively that all the possibilities in Proposition 3.1 in fact occur, and now we establish some results and notation that are needed for that. If $f, h \in$ $k[X, Y]$, we denote by $\operatorname{Res}_{X}(f, h)$ the resultant of $f$ and $h$ as elements of $(k[Y])[X]$ and $\operatorname{Res}_{Y}(f, h)$ denotes the resultant of $f$ and $h$ as elements of $(k[X])[Y]$.

LEMMA 3.2. Let $P=\left(a_{0}, \ldots, a_{2 m-n+2}, b_{0}, \ldots, b_{2 n-m+2}\right)$ be a point of $k^{n+m+4}$, and let $p(X)=\sum_{i=0}^{2 m-n+2} a_{i} X^{2 m-n+2-i}, q(X)=\sum_{j=0}^{2 n-m+2} b_{j} X^{2 n-m+2-j}$, and $f(X)=p(X) Y^{3}+$ $q(X)$. Suppose that $p$ and $q$ do not have common roots neither multiple roots. Then the set of points $P$ such that $\operatorname{Res}_{X}\left(\operatorname{Res}_{Y}(f, \partial f / \partial X), \operatorname{Res}_{Y}(f, \partial f / \partial Y)\right) \neq 0$ contains an open set of $k^{m+n+4}$.

Proof. We have $\operatorname{Res}_{Y}(f, \partial f / \partial Y)=27 p^{3} q^{2}$ and $\operatorname{Res}_{Y}(f, \partial f / \partial X)=p^{3}(d q / d X)^{3}-$ $3 q p^{2}(d q / d X)^{2}(d p / d X)+3 q^{2} p d q / d X(d p / d X)^{2}-(d p / d X)^{3} q^{3}$. From the hypothesis on $p(X)$ and $q(X)$, we conclude that if $f$ is such that $\operatorname{Res}_{Y}(f, \partial f / \partial X) \not \equiv 0$, then there is no $a \in k$ satisfying both $\operatorname{Res}_{Y}(f, \partial f / \partial Y)(X)=0$ and $\operatorname{Res}_{Y}(f, \partial f / \partial X)(X)=0$, i.e., $\operatorname{Res}_{X}\left(\operatorname{Res}_{Y}(f, \partial f / \partial X), \operatorname{Res}_{Y}(f, \partial f / \partial Y)\right) \neq 0$. Calculating $\operatorname{Res}_{Y}(f, \partial f / \partial X)$, we get

$$
\begin{aligned}
\operatorname{Res}_{Y}\left(f, \frac{\partial f}{\partial X}\right)= & (n-m)^{3} A_{0} X^{3 m+3 n+9}+(n-m)^{2} A_{1} X^{3 m+3 n+8} \\
& +(n-m) A_{2} X^{3 m+3 n+7}+A_{3} X^{3 m+3 n+6}+\cdots,
\end{aligned}
$$


where the dots indicate the sum of powers of $X$ lesser than $3 m+3 n+6, A_{0}=27 a_{0}^{3} b_{0}^{3}$ and $A_{3}$ is a polynomial expression in $a_{0}, b_{0}, a_{1}, b_{1}, a_{2}, b_{2}, a_{3}, b_{3}, m$, and $n$. More specifically, $A_{3}$ is a linear combination over $k$ of monomials of the type $a_{\alpha} a_{\beta} a_{\gamma} b_{\delta} b_{\epsilon} b_{\phi}$, with $\alpha, \beta, \gamma, \delta, \epsilon, \phi \in\{0,1,2,3\}$ and $\alpha+\beta+\gamma+\delta+\epsilon+\phi=3$, and calculating the coefficient of $a_{1}^{3} b_{0}^{3}$, we get $(3 n-3 m+1)^{3}$. So, $\operatorname{Res}_{Y}(f, \partial f / \partial X) \not \equiv 0$ for $P$ in an open set of $k^{n+m+4}$.

REMARK 3.3. Let $C_{0} \subset k^{2}$ be a nonsingular plane curve, defined by the equation $f_{0}(X, Y)=c_{\ell}(X) Y^{\ell}+\cdots+c_{0}(X)$, and let $C$ be the curve in $S_{m n}$, obtained by taking the closure in the Zariski topology of the image of $C_{0}$ in $U_{0}$ under the parametrization $(a, b) \mapsto\left(a^{0}: \cdots: a^{n}: a^{0} b: \cdots: a^{m} b\right)$. In [4, p. 67], it was shown that if $\ell>0$ and $C$ is nonsingular, then $C$ is also irreducible. This is done by considering that if $C$ has more than one (nonsingular) component, then they cannot intersect one another, but this is contradicted if one calculates their intersection numbers.

Proposition 3.4. Let $\sigma, \rho, \eta$, and $\xi$ be nonnegative integers satisfying the conditions in any of the items in Proposition 3.1, and let $m \leq n$ be positive integers such that $(n+2) / 2 \leq m \leq 2 n+2$ and $m+n+2 \leq 5$. Then there exists a trigonal nonsingular irreducible curve $C \subset S_{m n}$ of genus $g=m+n+2$ such that, in $C \cap E$, there are exactly $\sigma$ total ramification points and $\rho$ ordinary ramification points of $C$ and, in $C \cap D$, there are exactly $\xi$ total ramification points and $\eta$ ordinary ramification points of $C$.

Proof. We begin by assuming that $\sigma, \rho, \eta$ and $\xi$ satisfy the conditions (i) in Proposition 3.1. Let $r, s, u, v, a, b, c$, and $d$ be nonzero polynomials of degrees $\sigma, \rho$, $\eta, \xi, 2 m-n-\sigma-\rho, m+2-\sigma-\rho-\eta, n+2-\sigma-\eta-\xi$, and $2 n-m+2-\xi-\eta$, respectively, and let $f=r s a Y^{3}+r s u b Y^{2}+r u v c Y+u v d$. Let $C_{0}$ be the plane curve, defined by $f(X, Y)=0$, and let $C$ be Zariski closure in $S_{m n}$ of the curve in $U_{0} \stackrel{\sim}{\rightarrow} k^{2}$ that corresponds to $C_{0}$. We impose a series of open conditions on the coefficients of the polynomials $r, s, u, v, a, b, c$, and $d$ to grant that $C$ is a nonsingular curve. We begin by assuming that these polynomials do not have a common root when taken two at a time and that none of them have a multiple root. We also assume that the coefficient of $X^{2 m-n+1}$ in the polynomial $r s a$ is different from zero. We have

$$
\begin{aligned}
\operatorname{Res}_{Y}\left(f, \frac{\partial f}{\partial Y}\right)= & 27 a^{3} d^{2} r^{3} s^{3} u^{2} v^{2}-18 a^{2} b c d r^{4} s^{3} u^{3} v^{2}-a b^{2} c^{2} r^{5} s^{3} u^{4} v^{2} \\
& +4 a^{2} c^{3} r^{5} s^{2} u^{3} v^{3}+4 a b^{3} d r^{4} s^{4} u^{4} v
\end{aligned}
$$

Thus, $\operatorname{deg} \operatorname{Res}_{Y}(f, \partial f / \partial Y) \leq 4 m+n+10$ and we assume that the coefficients of the polynomials $r, s, u, v, a, b, c$, and $d$ are such that $\operatorname{Res}_{Y}(f, \partial f / \partial Y)$ is a polynomial in $X$ of degree $4 m+n+10$. Observe that if we substitute $b \mapsto 0$ and $c \mapsto 0$, then

$$
\operatorname{Res}_{Y}\left(\left.f\right|_{b=0, c=0},\left(\frac{\left.\partial f\right|_{b=0, c=0}}{\partial Y}\right)\right)=\left.\operatorname{Res}_{Y}\left(f, \frac{\partial f}{\partial Y}\right)\right|_{b=0, c=0}=27(r s a)^{3}(u v d)^{2}
$$

has also degree $4 m-n+10$ as a polynomial in $X$. Similarly, we assume that the degrees of $\operatorname{Res}_{Y}(f, \partial f / \partial X)$ and $\operatorname{Res}_{Y}\left(\left.f\right|_{b=0, c=0}, \partial\left(\left.f\right|_{b=0, c=0}\right) / \partial X\right)$ as polynomials in $X$ are equal to $3 m+n+9$ if $n \neq m$ or equal to $3 m+n+6$ if $n=m$ (as in the proof of Lemma 3.2, one may check that the coefficient of $X^{3 m+3 n+9}$ in $\operatorname{Res}_{Y}(f, \partial f / \partial X)$ is equal 
to $(n-m)$ times a nonidentically null polynomial expression in the leading coefficients of $r, s, u, v, a, b, c$, and $d$ that does not vanish when $b=c=0$; the coefficients of $X^{3 m+3 n+8}$ and $X^{3 m+3 n+7}$ are zero if $n=m$ and the coefficient of $X^{3 m+3 n+6}$ is a nonzero polynomial expression in $n, m$ and the coefficients of $r, s, u, v, a, b, c$, and $d$ that does not vanish when $n=m$ or when $b=c=0$ ).

Thus, we have

$$
\begin{aligned}
& \left.\operatorname{Res}_{X}\left(\operatorname{Res}_{Y}\left(f, \frac{\partial f}{\partial Y}\right), \operatorname{Res}_{Y}\left(f, \frac{\partial f}{\partial X}\right)\right)\right|_{b=0, c=0} \\
& =\operatorname{Res}_{X}\left(\operatorname{Res}_{Y}\left(\left.f\right|_{b=0, c=0}, \partial \frac{\left(\left.f\right|_{b=0, c=0}\right)}{\partial Y}\right), \operatorname{Res}_{Y}\left(\left.f\right|_{b=0, c=0}, \partial \frac{\left(\left.f\right|_{b=0, c=0}\right)}{\partial X}\right)\right) .
\end{aligned}
$$

Of course, we also substitute $d b / d x \mapsto 0$ and $d c / d x \mapsto 0$ in the expression on the left side of the equality. This equality together with Lemma 3.2 show that we may choose coefficients for $r, s, u, v, a, b, c$, and $d$ such that $\operatorname{Res}_{X}\left(\operatorname{Res}_{Y}(f, \partial f / \partial Y)\right.$, $\left.\operatorname{Res}_{Y}(f, \partial f / \partial Y)\right) \neq 0$, which implies that $C_{0}$, and a fortiori $C \cap U_{0}$ do not have a singular point. Observe that $C \cap L_{\infty} \cap E=\phi$ since $\operatorname{deg}(r s a)=2 m-n+2$. So, to grant the smoothness of the points of $C$ in $C \cap L_{\infty}$, it suffices to grant the smoothness of the points with zero abscissa of the plane curve defined by $f_{n}(X, Y)=0$, where $f_{n}(X, Y)$ is obtained from $f_{0}(X, Y)$ by substituting $X \mapsto 1 / X$ and multiplying the resulting coefficient of $Y^{i}$ by $X^{2 n-m+2-i(n-m)}, i=0,1,2,3$. To this end, it suffices to have $\operatorname{Res}_{Y}\left(f_{n}, \partial f_{n} / \partial Y\right)(0) \neq 0$ but this is just another open condition on the (leading) coefficients of $r, s, u, v, a, b, c$, and $d$, and so we may take it for granted. Finally, we should check the smoothness of the points of $C$ in $C \cap\left(E \backslash L_{\infty}\right)$ or, equivalently, we must check the smoothness of the points of zero ordinate of the plane curve defined by $f_{n+1}(X, Y):=u v d Y^{3}+r u v c Y^{2}+r s u b Y+r s a=0$. But this is already granted because, since $r s a$ is a polynomial without multiple roots, the curves defined by $f_{n+1}(X, Y)=0$ and $\left(\partial f_{n+1} / \partial X\right)(X, Y)=0$ have no points with zero ordinate in common. This shows that $C$ is a nonsingular curve and, by Remark 3.3, $C$ is an irreducible (trigonal, of genus $m+n+2$ ) nonsingular curve. Using Remark 2.1, it is easy to see that $C$ has exactly $\sigma$ (respectively, $\rho$ ) total (respectively, ordinary) ramification points lying over $E$ and $\eta$ (respectively, $\xi$ ) total (respectively, ordinary) ramification points lying over $D$.

To find curves $C$ with ramification points as described in the other items of Proposition 3.1 we proceed exactly as above, except for the starting curve $C_{0}$. If $\sigma$, $\rho, \xi$, and $\eta$ satisfy condition (ii) in Proposition 3.1, then we begin with a plane curve defined by $f(X, Y)=r s Y^{3}+r u c Y+u d$, where $r, s, u, c$, and $d$ are polynomials in $X$ of degrees $\sigma, \rho, \eta, n+2-\sigma-\eta$, and $2 n-m+2-\eta$ respectively. If $\sigma, \rho, \xi$, and $\eta$ satisfy conditions (iii) in Proposition 3.1, then we begin with a plane curve defined by $f(X, Y)=r a Y^{3}+r u b Y^{2}+u v$, where $r, u, v, a$, and $b$ are polynomials in $X$ of degrees $\sigma, \eta, \xi, 2 m-n+2-\sigma$, and $m+2-\sigma-\eta$ respectively. If $\sigma, \rho$, $\xi$, and $\eta$ satisfy conditions (iv) in Proposition 3.1, then we begin with a plane curve defined by $f(X, Y)=r Y^{3}+u$, where $r$ and $u$ are polynomials in $X$ of degrees $2 m+n-2$ and $2 n-m+2$ respectively.

Observe that the above proposition and the Riemann-Hurwitz Theorem show that 
there are trigonal curves $C$ such that all the points in $C \cap D$ or in $C \cap E$ are total ramification points and these are all the ramification points of $C$.

The next proposition extends a result in [2], where similar bounds were obtained but only total ramification points were considered.

Proposition 3.5. Let $\sigma$ (respectively, $\rho$ ) be the number of total (respectively, ordinary) ramification points on E. Let $\tau$ (respectively, $\varsigma$ ) be the number of total (respectively, ordinary) ramification points in $C$. Then one of the following possibilities occurs.

(i) $\tau=2 n-m+2, \sigma=2 m-n+2$ and $C$ has no other ramification points.

(ii) $0 \leq \tau+\varsigma+2 \sigma+\rho \leq 2 m+4$.

Proof. Let $f_{0}=c_{3}(X) Y^{3}+c_{2} Y^{2}+c_{1}(X) Y+c_{0}(X)$ be the equation of the plane curve $C_{0}$ which is isomorphic to $C \cap U_{0}$, as we have seen in the last section, and suppose that $\operatorname{Res}_{Y}\left(\partial f_{0} / \partial Y, \partial^{2} f_{0} / \partial Y^{2}\right) \neq 0$. It is easy to check that if $(a, b)$ is a total ramification point of $C_{0}$, then $a$ is a root of $12 c_{3}(X)\left(3 c_{1}(X) c_{3}(X)-c_{2}(X)^{2}\right)=$ $\operatorname{Res}_{Y}\left(\partial f_{0} / \partial Y, \partial^{2} f_{0} / \partial Y^{2}\right)$ and Remark 2.1(i) yields that $a$ must be a root of $3 c_{1}(X) c_{3}(X)$ $-c_{2}(X)^{2}$. From the bounds on the degrees of $c_{1}, c_{2}$, and $c_{3}$, we see that this polynomial has a degree which is at most $2 m+4$. From Remark 2.1(iv), we have that if $\left(a^{\prime}, 0\right)$ is an ordinary (respectively, a total) ramification point of $C_{n+1}$, then $a^{\prime}$ is a root (respectively, is at least a double root) of $3 c_{1}(X) c_{3}(X)-c_{2}(X)^{2}$. In a similar way, one may see that if there is a point of the form $(0, b)$ in $C_{n}$ and it is a total ramification point, then 0 is a root of $3 \tilde{c}_{1}(X) \tilde{c}_{3}(X)-\tilde{c}_{2}(X)^{2}=\left(3 c_{1}(1 / X) c_{3}(1 / X)-C_{2}(1 / X)^{2}\right) X^{2 m+4}$. Also, if the point at the origin of $k^{2}$ is an ordinary (respectively, a total) ramification point of $C_{g-1}$, then, by Remark 2.1(v), we must have that 0 is a root (respectively, is at least a double root) of $3 \tilde{c}_{1}(X) \tilde{c}_{3}(X)-\tilde{c}_{2}(X)$. Note that these two last cases are mutually exclusive, for the points in the $Y$-axis of the plane that contains $C_{g-1}$ as well as the points in the $Y$-axis of the plane that contains $C_{n}$ are all mapped into the line $L_{\infty}$ through the appropriate local parametrizations of $S_{m n}$ described in the previous section and of course a line in the scroll may contain at most one ramification point of $C$. Moreover, for one of these two last cases to occur, we must have $\operatorname{deg}\left(3 c_{1}(X) c_{3}(X)-c_{2}(X)^{2}\right)<2 m+4$ so that anyway, we get $0 \leq \tau+2 \sigma+\rho \leq 2 m+4$.

Suppose now that $3 c_{1}(X) c_{3}(X)-c_{2}(X)^{2}=0$. Then by Remarks 2.1(iv) and 2.3, we see that $c_{3}(X)$ does not have a multiple root and that $c_{3}(X) \mid c_{1}(X)$. Observe that, by Remark 2.2, we must have $\operatorname{deg} c_{3}(X)=2 m-n+2, \operatorname{deg} c_{2}(X)=m+2$, and $\operatorname{deg} c_{1}(X)=$ $n+2$ or else $\operatorname{deg} c_{3}(X)=2 m-n+1, \operatorname{deg} c_{2}(X)=m+1$, and $\operatorname{deg} c_{1}(X)=n+1$. Either way, from Remark 2.1, we have that $\sigma=2 m-n+2$ and $\rho=0$. To establish the first assertion now, we use the Riemann-Hurwitz Theorem and note that it is enough to show that there is no ordinary ramification point of $C$ in $C \backslash E$. From $3 c_{1}(X) c_{3}(X)=c_{2}(X)^{2}$, it is easy to verify that $12 c_{3}(X)\left(\partial f_{0} / \partial Y\right)(X, Y)=\left(\partial^{2} f_{0} / \partial Y^{2}\right)^{2}(X, Y)$ and also that there is no ordinary ramification point in $C_{0}$. Finally, we note that $3 \tilde{c}_{1}(X) \tilde{c}_{3}(X)=\tilde{c}_{2}(X)^{2}$, hence, $12 \tilde{c}_{3}(X)\left(\partial f_{n} / \partial Y\right)(X, Y)=\left(\partial^{2} f_{n} / \partial Y^{2}\right)^{2}(X, Y)$. Then, as above, we conclude that there is no ordinary ramification point in $C_{n}$, which completes the proof.

ACKNOWLEDGement. This work was done during a visit to the Department of Mathematics of Harvard University. I would like to thank the staff and faculty of that department for the kind reception specially Prof. Joseph Harris for the invitation. 


\section{REFERENCES}

[1] A. Andreotti and A. L. Mayer, On period relations for Abelian integrals on algebraic curves, Ann. Scuola Norm. Sup. Pisa (3) 21 (1967), 189-238. MR 36\#3792. Zbl 222.14024.

[2] M. Coppens, The Weierstrass gap sequences of the total ramification points of trigonal coverings of $\mathbb{P}^{1}$, Nederl. Akad. Wetensch. Indag. Math. 47 (1985), no. 3, 245-276. MR 87c:14028. Zbl 592.14025.

[3] T. Kato and R. Horiuchi, Weierstrass gap sequences at the ramification points of trigonal Riemann surfaces, J. Pure Appl. Algebra 50 (1988), no. 3, 271-285. MR 89d:14045. Zbl 649.14009.

[4] K.-O. Stöhr and P. Viana, Weierstrass gap sequences and moduli varieties of trigonal curves, J. Pure Appl. Algebra 81 (1992), no. 1, 63-82. MR 93f:14017. Zbl 768.14016.

Carvalho: Departmento de Matemática, Universidade Federal de Uberlândia, Av. UNIVERSITÁRIA S/N. 38400 UBERLÂNDIA-MG, BRAZIL

E-mail address: cicero@impa.br 


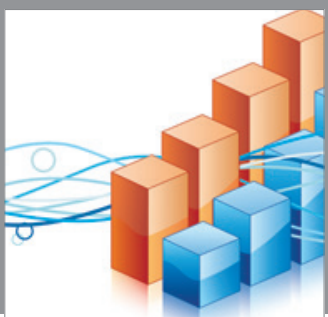

Advances in

Operations Research

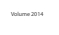

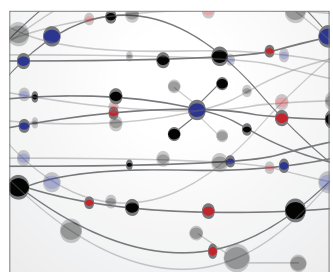

\section{The Scientific} World Journal
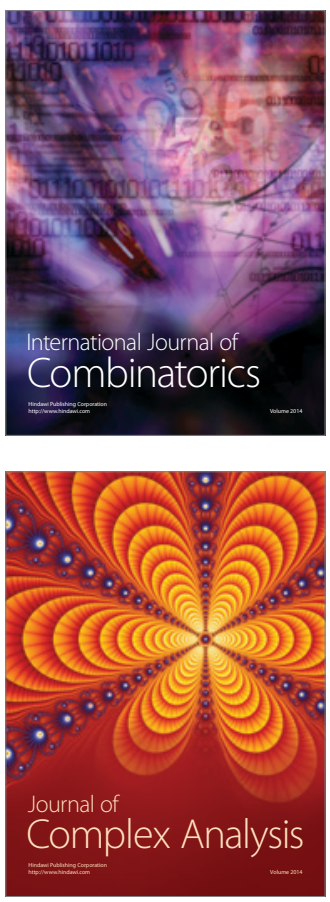

International Journal of

Mathematics and

Mathematical

Sciences
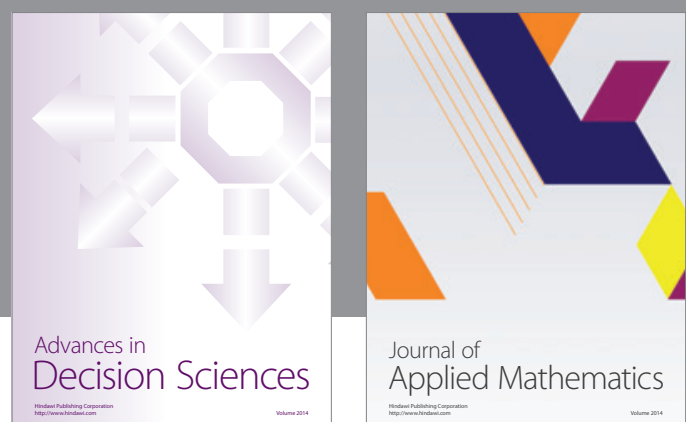

Journal of

Applied Mathematics
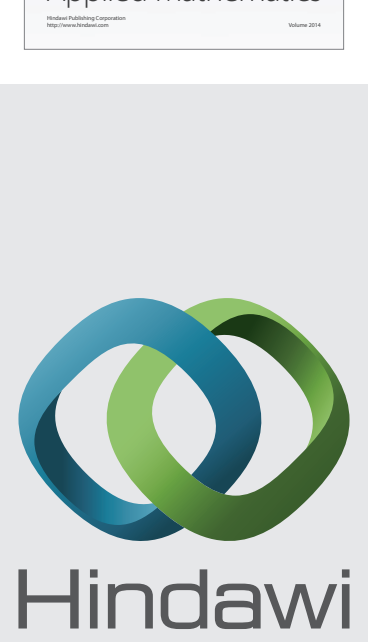

Submit your manuscripts at http://www.hindawi.com
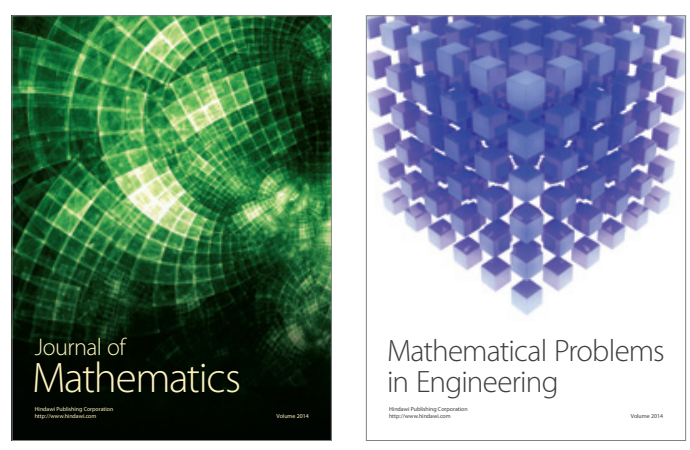

Mathematical Problems in Engineering
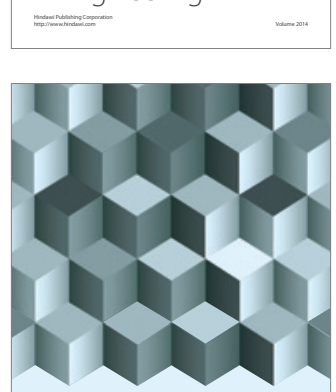

Journal of

Function Spaces
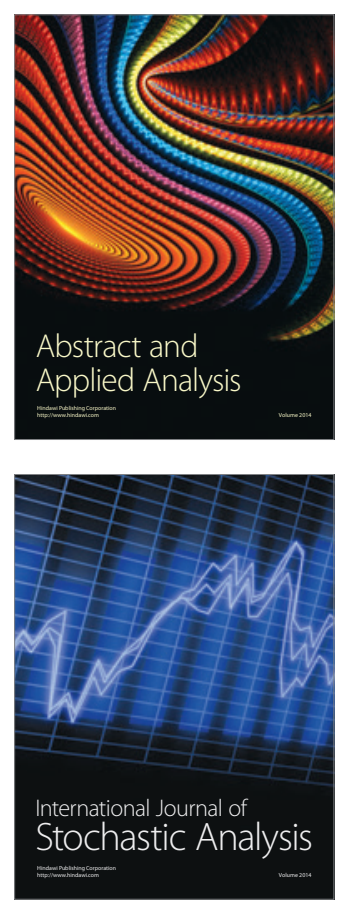

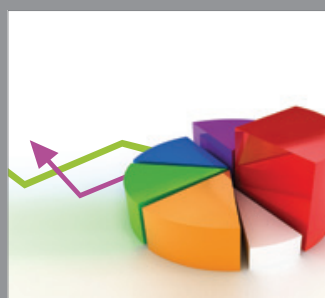

ournal of

Probability and Statistics

Promensencen
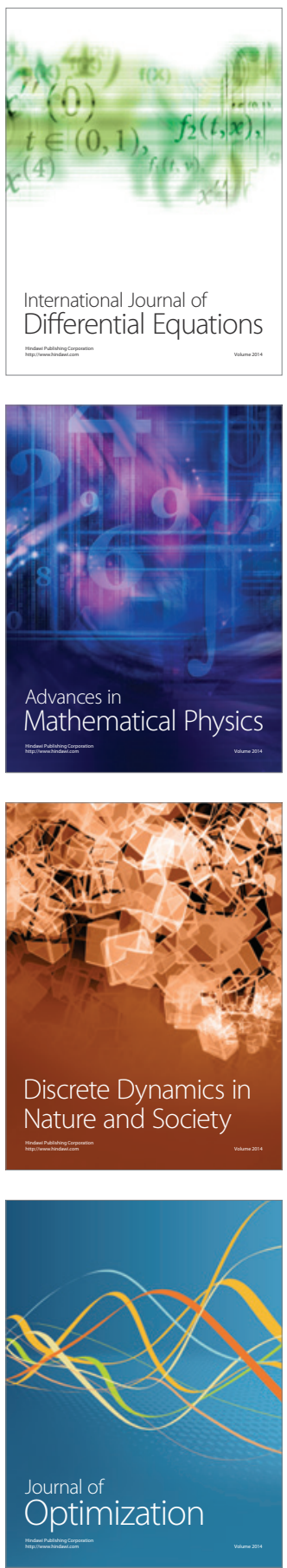